\title{
Discovering Configuration Templates of Virtualized Tenant Networks in Multi-tenancy Datacenters via Graph-mining
}

\author{
Yosuke Himura \\ Hitachi Yokohama Research Laboratory
}

\author{
Yoshiko Yasuda \\ Hitachi Yokohama Research Laboratory
}

\begin{abstract}
Multi-tenant datacenter networking, with which multiple customer (tenant) networks are virtualized over a single shared physical infrastructure, is cost-effective but poses significant costs on manual configuration. Such tasks would be alleviated with configuration templates, whereas a crucial difficulty stems from creating appropriate (i.e., reusable) ones. In this work, we propose a graph-based method of mining configurations of existing tenants to extract their recurrent patterns that would be used as reusable templates for upcoming tenants. The effectiveness of the proposed method is demonstrated with actual configuration files obtained from a business datacenter network.
\end{abstract}

\section{Categories and Subject Descriptors}

C.2.3 [Network Operations]: Network management

\section{General Terms}

Algorithms, Experimentation, Management, Measurement

\section{Keywords}

Datacenter network, Network configuration, Configuration analysis, Multi-tenancy, Graph-mining, Template

\section{INTRODUCTION}

There has been a significant rise in the utilization of multitenancy in enterprise datacenter networking. Multi-tenancy is a form of deploying customer (tenant) networks, with which multiple customer networks are virtualized and consolidated in a single shared physical network and yet logicallyindependent each other $[1,2]$. Leveraging multi-tenancy is hence cost-effective, being enabled by recent technologies of network virtualization; In addition to the conventional L2-level separation with virtual LAN (VLAN), L3-level separation is allowed by the use of virtual interface (VIF) and virtual router (VR). According to the recent increase in the processing performance of network devices, tens or even hundreds of tenants can be deployed in a single datacenter.

One of the essential tasks in multi-tenant datacenters is to create network configuration settings for deploying tenants, which are diversely composed according to various enterprise requirements (e.g., multi-tier architecture). Tenant construction in such datacenters still mostly relies on manual configuration because of the lack of tools that generally meet those various requirements. Manual configuration is substantially time-consuming and error-prone in general as claimed over at least a decade $[3,4,5,6,7]$, and the use of multi-tenancy in datacenter context has unfortunately required more careful and sophisticated management processes than ever due to the increase in risks of misconfigurations in such shared environments.

A conventional approach for mitigating the burden of manual configuration is to leverage configuration templates. A configuration template is defined as a predefined composition of setting commands without specific assigned parameters (e.g., VLAN ID), which are automatically or manually assigned at the time of deployment. The use of templates is effective when similar form of configurations are repetitively created (i.e., templates are reusable). According to successful cases in other areas (e.g., ISP $[4,6,7]$ ), we expect that templates would also be effective in multi-tenancy datacenter networks.

A crucial problem in the use of templates, however, stems from the difficulty in creating appropriate (i.e., reusable) ones. Indeed, there is no generally-applicable templates, because the composition of tenant configurations varies depending on the form of physical networks (e.g., device types) and on specific domain-knowledge of network engineers. In addition, finding appropriate templates is uneasy as the frequent patterns are a priori unknown.

In this work, we aim to automatically generate appropriate configuration templates for deploying tenants in multitenant datacenters. The main idea towards this goal is derived from mining configurations of existing (i.e., alreadydeployed) tenants to find typical patterns of them, which would be represented as reusable templates for upcoming tenants. We propose a method based on (a) an abstraction of tenant configuration as attributed graphs, which enables to measure similarity among them, and on (b) an unsupervised clustering over those attributed graphs, which produces groups of similar (i.e., recurrent form of) tenants. The proposed method is evaluated with actual configuration files obtained from a multi-tenant production datacenter. This evaluation demonstrates that the proposed method correctly finds typical patterns of tenant configurations that can be effectively used as configuration templates for upcoming tenants. Originalities of the present work include (1) the method of automatically finding typical patterns of tenant configurations for creating appropriate templates, and (2) the case-study with an actual production datacenter.

\section{BACKGROUND AND MOTIVATION}

We first present the basic construction of the physical network in a datacenter (Sec. 2.1) and how network devices are 


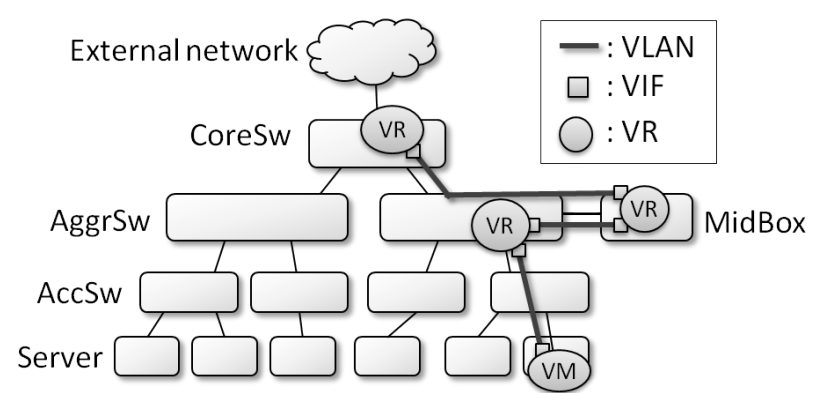

Figure 1: Schematic representation of a conventional physical network of datacenter deploying a virtual network for a tenant.

configured in order to deploy virtualized networks for tenants over the datacenter (Sec. 2.2). Then, we explain the problem of difficulty in creating configurations (Sec. 2.3).

\subsection{Multi-tenancy datacenter network}

Figure 1 illustrates a conventional datacenter network. Typical datacenters comprise following devices.

- Access switches (AccSw) connect multiple servers basically with L2 functionalities.

- Aggregation switches (AggrSw) aggregate access switches and connect middle-boxes, providing L3 connectivity.

- Middle-boxes (MidBox) provide network services such as firewall filtering, load balancing, NAT, VPN termination, or SSL acceleration.

- Core switches (CoreSw) further merge traffic from aggregation switches if necessary and connect to external networks (e.g., enterprise networks, or the Internet).

We note that datacenter is usually constructed as redundancy structure to achieve high availability, although we omitted this from the figure for readability.

This single infrastructure consolidates separated virtual networks for multiple customers, which we define as tenant networks. Figure 1 also illustrates a tenant network deployed over the above-mentioned physical devices. Engineers use following virtualization technologies to achieve logical independence among different tenant networks.

- Virtual Router (VR) generates logical instances for routing functionality inside a device. A representative of VR used in many networking fields is known as Virtual Routing and Forwarding (VRF).

- Virtual Interface (VIF) creates logical instances of network interface. Well-known realizations of VIF are VLAN interface and sub-interface.

- Virtual LAN (VLAN) (mainly IEEE 802.1Q) is a common technology for L2 separation, which virtually separates a physical link into logically independent links.

These virtualization technologies create logical instances of network components such as routers, interfaces, or links we name them virtual resources, and hence a tenant network can be interpreted as a collection of virtual resources.

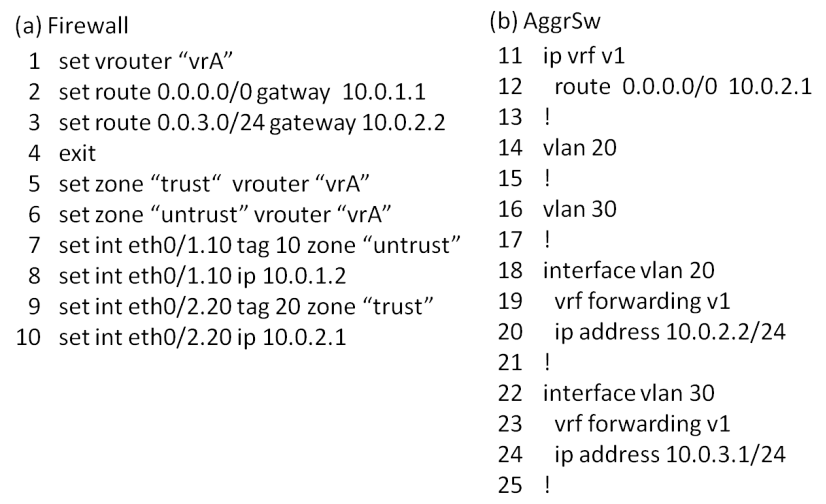

Figure 2: Configuration commands of a firewall MidBox (left) and an AggrSw (right) for the tenant network depicted in Figure 1.

\subsection{Tenant configuration}

Configuration settings in the networking literature are generally stored as configuration files, each of which is a list of commands of networking functions. Figure 2 represents two examples of configuration files used to construct a tenant network. Here, Figure 2(a) and (b) correspond to a list of configuration commands for a firewall as MidBox role and a switch as AggrSw role, respectively. Those configuration files are related to the tenant network depicted in Figure 1. We note that these configuration files are partially modified for brevity and not exactly same as those of existing products.

These configuration files can be broken down in terms of virtual resources as follows.

- Allocating VR instances and specifying static routes (lines 1-4, 11-13): The virtual router named vrA is allocated in the firewall and that tagged as v1 is allocated in the switch.

- Creating VIF instances and attaching them to a VR instance (lines 5-10, 18-25): For instance, the firewall creates two VIFs named eth0/1.10 and eth0/2.20, and connect these interfaces with the virtual router vrA via the definition of zones, each of which is a logical grouping of interfaces. These lines include the assignment of IP address on the interfaces; IP addresses on VIFs are essential for routing among multiple VLANs.

- Defining VLAN instances and relating them to VIFs (lines 7, 9, 14-17, 18, 22): Some lines can be interpreted as creating VLAN instances from the viewpoint of virtual resources, although they are not explicitly declared. For example, lines 7 and 9 can be interpreted as creating VLANs 10 and 20 and attaching them to the physical interfaces eth0/1.10 and eth0/2.20.

Our empirical observation on real enterprise datacenters was that tens or even a hundred of tenants are contemporarily consolidated in a single shared infrastructure, resulting in thousands (or even tens of thousands) of lines of commands.

\subsection{Problem in deploying tenants}

Usual procedure of deploying a tenant has mostly been conducted in a manual fashion due to the lack of tools suitable for the variety of requirements for enterprise networks 


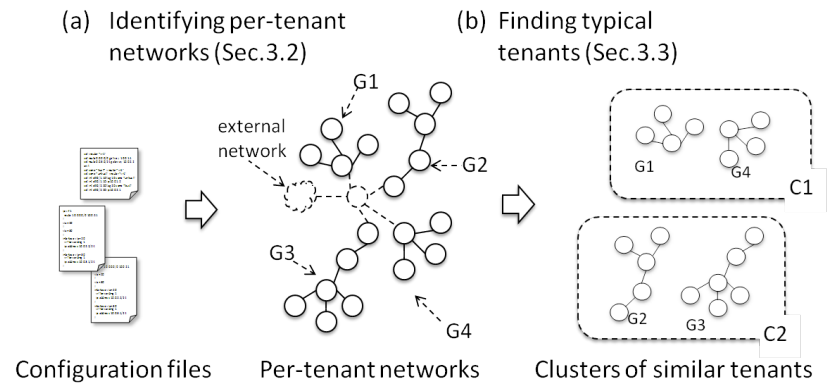

Figure 3: Overview of the proposed method.

(e.g., multi-vendor infrastructure, multi-tier tenant topology). Manual configuration is notoriously time-consuming and error-prone as claimed over at least a decade.

The use of template would alleviate the burden of manual configuration in general. Templates are effective as long as there are recurrent patterns of provisioned networks because of their reusability. We empirically observed such recurrent patterns in multi-tenancy environments, and hence we consider that this general approach would be applicable as well.

A crucial problem in the use of templates, however, is the difficulty in creating appropriate set of configuration templates. We regard the appropriateness of templates as their reusability, and thus such templates should reflect the frequent patterns of tenant configuration; However, such frequent patterns are generally a priori unknown.

\section{METHODOLOGY}

\subsection{Overview}

We aim to automatically generate appropriate (i.e., reusable) configuration templates for tenant deployment in multi-tenant datacenters. In the present work, we focus on the topologies of tenants as the first step toward this goal, because most of networking functions such as routing, filtering, and/or quality-assuring are realized after constructing network topology. The main idea for auto-finding topology templates is based on mining existing tenant network configurations to discover recurrent patterns of their topologies that can be repeatedly used as templates for upcoming tenants. We develop an automated method, which is represented as Figure 3, consisting of two major steps: (a) the identification of per-tenant networks by analyzing configuration files and abstraction of them as attributed graphs (Sec. 3.2), and (b) the discovery of recurrent patterns of tenant networks based on a graph mining technique performed over the abstracted tenant networks (Sec. 3.3).

\subsection{Identifying tenant networks}

The first task is to identify per-tenant network topology as there is no direct mapping between a tenant network and a device configuration. We first present the model of tenant network (Sec. 3.2.1) and the method of acquiring them from configuration files (Sec. 3.2.2).

\subsubsection{Tenant network model}

Since a tenant network consists of a set of virtual resources and connections between pairs of them as described in Sec. 2.2, any tenant network can be abstracted as an undirected graph; A node (vertex) represents an instance of virtual resource (i.e., VR, VIF, VLAN), whereas a single edge stands for the referential relationship between a pair of virtual resources. This device-neutral abstraction allows us to leverage a variety of analytical capabilities for graphs.

We formally define a tenant topology as an attributed graph $G=(V, E, \Sigma)$, where $V=\left(v_{1}, \ldots, v_{N}\right)$ is a set of nodes, $E=\left(e_{1}, \ldots, e_{M}\right)$ is a set of edges (also noted as $\left.e_{i}=\left(v_{j}, v_{k}\right)\right)$, and $\Sigma: V \rightarrow L$ is a labeling function that maps a node to a label. A label $l \in L$ is composed of 4 attributes $l=(t, p, d, r)$, where $t$ is the type of virtual resource (e.g., VLAN, VR), $p$ is the parameter of virtual resource (e.g., 10, eth0/2.20, vrA), $d$ is the ID of device, and $r$ is the role of device (e.g., coreSw, aggrSw). For example, a virtual router vr3 in core switch CS1 is abstracted as a node $v_{i}$ with the corresponding label $L\left(v_{i}\right)=(t, p, d, r)=$ $(V R, v r 3, C S 1$, core $S w)$. The first 3 attributes $(t, p, d)$ are required for uniquely identifying the virtual resource among others in the network, whereas we use 4 th one $(r)$ for the configuration mining as shown in Sec. 3.3.

We note that the device id $(d)$ and the device roll $(r)$ of VLAN node should exceptionally be constant across all the devices (e.g., $d=n u l l$ ), because we regard a VLAN node as an instance of an aggregated L2 network connecting multiple virtual interfaces across devices. This abstraction of an L2 network as a node hides the need for inputting physical topology to obtain correct topology, which can be conducted when networks are correctly configured; This would be true in the in-service datacenter, where deployed tenants are actually and correctly providing services.

We also note that the definition of the graph $G$ does not contain virtual hosts (VMs) as nodes, but contain only virtual networking resources (e.g., VLAN, VR). The main motivation for this is that the number of VMs can easily be various according to the computational requirement from tenants, compared to that of networking nodes. Even if a pair of graphs have the identical network configuration, those graphs would produce high dissimilarity if they contained different number of VMs as nodes, impairing the discovery of typical networking configurations.

\subsubsection{Acquiring tenants from configuration files}

We select to reverse-engineer configuration files to obtain tenant networks. This choice is motivated by the fact that those files (a) are commonly available for engaged network operators, (b) store correct running status of networks, and (c) can be automatically analyzed thanks to their predefined syntaxes. Identification of tenant topologies is, however, not obvious due to the inexistence of direct mapping between a configuration file and a tenant; A single tenant network is composed of several virtual resources allocated by several devices, whereas a single device allocates lots of virtual resources for multiple tenant networks.

Identification of tenant topologies from configuration files can be achieved according to the following two steps. (1) According to the tenant network model (Sec. 3.2.1), we first generate the nodes from all the configuration files, and then produce the edges connecting these nodes by re-scanning those files. (2) We use a conventional graph traversal method to obtain per-tenant networks appearing as 'connected components' inside the entire network reproduced by the above step (Figure 3(a)). This second step is plausible because any pair of tenant networks must not be inter-connected due to the principle of multi-tenant networking. 
Indeed, there might be a case where tenant networks are exceptionally inter-connected at a gateway router; We need to remove the corresponding node before performing the graph traversal in this case. Removal of the shared nodes can be both manual and automatic; Those nodes can manually be indicated by operators, or can automatically be identified based on heuristics (e.g., such shared node will have many edges than the others). In our case, we manually indicated the shared nodes beforehand, as they were a priori known.

\subsection{Finding typical tenants}

The second step after identifying tenant networks is to extract similar patterns of them. We breakdown this issue into defining similarity between a pair of topologies with graph edit distance customized for this specific issue (Sec. 3.3.1), and finding clusters of similar topologies with an unsupervised clustering (Sec. 3.3.2).

\subsubsection{Computing tenant similarities}

Discovering typical patterns can generally be achieved by unsupervised clustering, which groups similar objects on the basis of a quantified similarity measure. Conventional similarity is Euclidean distance between two vectors, but unfortunately this measure cannot be directly applied to structured graphs.

In contrast to the conventional approach, we leverage graph edit distance $D\left(G_{1}, G_{2}\right)$ to quantify the similarity between a pair of graphs $G_{1}$ and $G_{2}$. The graph edit distance is the minimum number of operations needed for transforming $G_{1}$ into an isomorphic of $G_{2}$ [8]. The transformational operations are addition/deletion of a single node/edge. Here, $G_{1}=\left(V_{1}, E_{1}, \Sigma_{1}\right)$ and $G_{2}=\left(V_{2}, E_{2}, \Sigma_{2}\right)$ are isomorphic if there is a bijective function $f: V_{1} \rightarrow V_{2}$ satisfying both (a) $\Sigma_{1}(v)=\Sigma_{2}(f(v))$ for $\forall v \in V_{1}$, and (b) $\left(f\left(v_{i}\right), f\left(v_{j}\right)\right) \in E_{2}$ for $\forall\left(v_{i}, v_{j}\right) \in E_{1}$. The isomorphism between a pair of graphs means that the two graphs have the same set of labeled nodes representing the identical structure. Higher $D\left(G_{1}, G_{2}\right)$ indicates higher degree of structural difference between $G_{1}$ and $G_{2}$.

In the context of discovering similar patterns of tenant networks, we decided that two nodes can be mapped via $f$ if their node types $t$ and device roles $r$ are same. In other words, this similarity does not consider the parameter of virtual resource $p$ and the ID of physical device $d$. The basic structure of tenant networks can be represented with $t$ and $r$, and thus assigned parameters $p$ and actual used devices $d$ should be determined at the moment of using a template (not the moment of creating templates). Hence instead of the labeling function $\Sigma$ (Sec.3.2.1), we define an alternate labeling function $\Sigma^{\prime}: V \rightarrow L^{\prime}$, where $l^{\prime} \in L^{\prime}$ consists of the 2 attributes $l^{\prime}=(t, r)$. With this similarity measure, we compute the $N \times N$ similarity matrix $A=\left(a_{i, j}\right)$ for $N$ graphs $G_{1}, \ldots, G_{N}$, where $a_{i, j}=D\left(G_{i}, G_{j}\right)$.

The main motivation to use the graph edit distance is that this metric directly reflects the engineering workload to create a tenant configuration settings. For example, adding a node to a graph is similar to the attaching a virtual resource (e.g., VR) with a tenant network. This engineering nature would not easily be represented by isomorphism or by statistical metrics such as eigenvalues, degree distributions, centrality measures (e.g., betweenness), and so on.

\subsubsection{Clustering tenant networks}

Unsupervised clustering produces clusters of graphs based on similarities between graphs. A collection of $N$ graphs $G_{1}, \ldots, G_{N}$ is converted into a set of $M$ clusters $C_{1}, \ldots, C_{M}$ (Figure 3(b)). A cluster $C_{i}$ is a collection of graphs that are close in the distance space, i.e., similar in terms of topological shape.

We use an aggromerative hierarchical clustering [9]. This technique starts with $N$ clusters, each of which contains a single graph, then a pair of most similar clusters are merged as one. The operation of merging clusters is iterated until any pair of clusters does not yield distance higher than a threshold $\theta$. Here, the distance of two clusters $D\left(C_{i}, C_{j}\right)$ is defined as $D\left(C_{i}, C_{j}\right)=E\left(C_{i} \cup C_{j}\right)-E\left(C_{i}\right)-E\left(C_{j}\right)$, where $E\left(C_{i}\right)$ is the Euclidean intra-cluster distance within $C_{i}$. The inter-cluster distance $D\left(C_{i}, C_{j}\right)$ can be computed from the similarity matrix $A$, following the Lance-Williams similarity update formula. Refer to Ref. [9] for details. Clusters obtained with $\theta=0$ include only exactly identical structure of graphs, whereas those with $\theta>0$ result from fuzzy matching among graphs.

\subsubsection{Computational load}

The computation of the graph edit distance is most expensive as it requires exponential time- and space-complexity. Computational costs we measured were less than $10 \mathrm{sec}$. with a $3.3 \mathrm{GHz} \mathrm{CPU}$ and $1 \mathrm{~GB}$ memory space for conventional graphs (around 10 through 30 nodes - most of the graphs examined in this work); Those for large graphs (around 100 nodes) were unfortunately intractable due to the large search space, but this is insignificant because such graphs are generally atypical in practice so that template finding does not require the exact computation of similarity regarding such highly-different graphs. One option for this issue is to use the isomorphism as an alternative similarity measure (e.g., 0 for isomorphic and 1 for non-isomorphic) instead of the graph edit distance for low computational cost at the sacrifice of fuzzy clustering. More specifically, the use of isomorphism corresponds to the $\theta=0$ case of the use of graph edit distance, and hence can only obtain clusters with $\theta=0$ as well as cannot provide interpretation of the degree of dissimilarity among different clusters.

On the other hand, the clustering method requires $O\left(N^{2}\right)$ with $N$ objects for both time- and space-complexity, and we observed that 1,000 objects are clustered within a few minutes with that $\mathrm{CPU}$, requiring $10 \mathrm{MB}$ memory space.

Overall, the total processing time we measured with the dataset described later was less than 3 hours (computation of intractable cases were not completed but terminated during the processing when reaching to the limitation of memory). Hence, in practice, this processing can daily be performed to follow the longitudinal changes in typical pattern of tenant networks.

\section{VALIDATION}

\subsection{Dataset}

The feasibility of the proposed method is demonstrated with a set of configuration files obtained from switches and firewalls in an actual business datacenter. This datacenter offers a hosting service that consolidates virtualized systems of various customers in a multi-tenancy fashion. More than 1,000 instances of virtual resources are combined to form a 


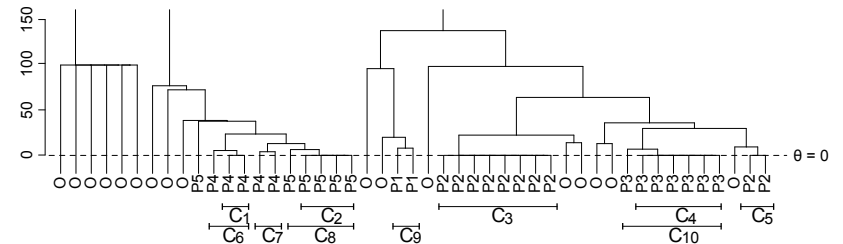

Figure 4: Clustering result represented as a hierarchical view with manually classified labels, showing the characteristics of the threshold $\theta$.

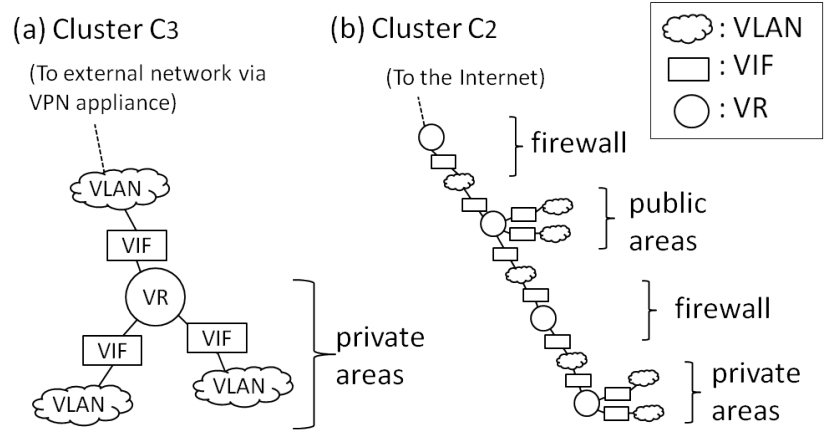

Figure 5: Examples of typical patterns found.

number of virtualized tenant networks. Those configuration files compose tens of thousands of lines in total.

In order to well interpret the results produced from the method, we create a referential information of tenant topologies by inspecting operational documents maintained by engineers dedicated to that datacenter. These documents consists of the drawing of topological structure of virtual resources allocated for each tenant. As a preliminary setup, we put effort on manually classifying around 50 tenants by examining their visual figures. This procedure identified 5 typical patterns based on their service usage. We annotate these typical patterns as P1 through P5 and atypical patterns as $\mathrm{O}$ (i.e., Other).

\subsection{Relevance of method}

Figure 4 displays the clustering result of tenant topologies. The horizontal line represents a series of the tenants (labeled by hand), whereas the vertical line measures the similarity between tenants (e.g., zero similarity means identical topology). We found that there were intractable similarity values for large graphs as discussed in Sec. 3.3.3. We decided to set the similarity 100 in that case as it is unimportant for template finding to exact computation of similarity regarding such highly-different graphs. Such topologies are located at the left area in the figure. Labeled clusters $C_{1}$ though $C_{5}$ are obtained with $\theta=0$, whereas $C_{6}$ through $C_{10}$ are produced with $\theta>0$.

This figure leads to the following findings.

- The resulting clusters having the same form of tenants imply that the use of template would be effective (i.e., reusable) in the examined environment. For example, with respect to the threshold $\theta=0$, there are 8 same topologies labeled as P2 in Cluster $C_{3}$, and 6 ones labeled as P3 in Cluster $C_{4}$. Approximately
$50 \%$ of tenants are clustered into $C_{1}$ through $C_{5}$ in total. Regarding the remaining half of tenants, some are clustered with $\theta>0$, but others (mainly labeled $O-$ about $35 \%$ ) are not clustered, as shown below.

- Also, this method successfully captured minor clusters containing small amount of tenants (e.g., $C_{1}, C_{5}$ ). Indeed, it should be easy to manually enumerate major clusters containing high number of tenants (e.g., $C_{3}$, $C_{4}$ - each of which contains over $10 \%$ of total tenants), because human can easily perceive frequently repetitive patterns from the entire set of tenants. On the other hand, however, manual enumeration will tend to overlook less frequent patterns; This difficulty will become polynomially significant according to the increase in the total number of tenants $N$, as finding typical tenants requires to compare each pair of two tenants, resulting in $N \times(N-1) / 2$ comparisons.

- Regarding the fuzzy clustering with $\theta>0$, we discovered several tenants that are similar yet not exactly identical (e.g., Clusters $C_{6}$ through $C_{10}$ ). We manually inspected their topologies and found that their difference mostly came from the number of network segments (VLANs) in a tenant. Such tenants would be constructed with small modification of configuration commands generated via templates, as the graph edit distance directly reflects the number of modification steps. For example, $C_{10}$ tenants can be created with small modification from $C_{4}$ configuration commands since $C_{4} \in C_{10}$. Also, we consider that the slight differences among the tenants within a fuzzy cluster can possibly be further parameterized, and such parameterization can be further effective in creating configurations; However, automatic extraction of parameterization is a difficult problem, which should be explored in the future work.

- On the other hand, there are a non-negligible amount of atypical tenants (labeled as $\mathrm{O}$ ), which are not similar to any of typical ones; Such tenants account for about $35 \%$ of total tenants as shown in Figure 4. Manual inspection on the composition of those tenants revealed that some of them consisted of tens of even around a hundred of virtual resources; Those tenants cannot be effectively represented as templates and should be manually customized as value-added services.

Figure 5 exhibits two of the typical patterns found by the proposed method.

- Figure 5(a) shows the most frequent pattern of tenant topology (Cluster $C_{3}$ ), which is composed of a single VR and a few VLANs. Consulting the operators' documents, we found that one of the VLANs is attached to a VPN device, which is used in establishing private connections between this datacenter and customer sites via external networks.

- On the other hand, Figure 5(b) displays another recurring pattern (Cluster $C_{2}$ ), which consists of two types of areas, i.e., public areas and private ones; The public areas are connected to the external network via a virtual router in a firewall, and to the private networks through another virtual router of firewall as well. This 
Table 1: Operational cost with and without templates based on auto-discovered patterns.

\begin{tabular}{|c|c|c|c|}
\hline & Operation & w/o template & w/ template \\
\hline \hline 1 & Topology design & $20 \mathrm{~min}$ & $20 \mathrm{~min}$ \\
\hline 2 & Configuration creation & $120 \mathrm{~min}$ & $\sim 0 \mathrm{~min}$ \\
\hline 3 & Self-validation & $60 \mathrm{~min}$ & $60 \mathrm{~min}$ \\
\hline 4 & Cross-validation & $60 \mathrm{~min}$ & $\sim 0 \mathrm{~min}$ \\
\hline \hline & Total & $240 \mathrm{~min}$ & $80 \mathrm{~min}$ \\
\hline
\end{tabular}

is a common construction of multi-tier network architecture with different security levels across tiers.

To summarize, we found that the proposed method fruitfully captured common usages of virtualized networks in a multitenant datacenter.

\subsection{Operational practicability}

The automated discovery of typical tenant configuration allowed us to implement a prototype of multi-tenancy-aware networking management tool, which automatically generates a set of configuration files for a tenant based on the 5 configuration templates corresponding to the discovered patterns $\mathrm{C} 1$ through $\mathrm{C} 5$ (can be obtained with $\theta=0$ ). The parameters of various types of resources (e.g., VLAN ID, VRF ID) are automatically assigned by the tool so as not to cause misconnection to any of the existing tenants. The users of this tool only have to select an appropriate template if it matches with the requirement from the upcoming customer. As shown in Sec. 4.2, about $50 \%$ of tenant networks can be represented as one of the 5 templates, although this service still accepts customized tenant networks.

An engineer actually used this tool. Usual procedure to deploy a tenant was as follows (Table 1): (1) designing the topological structure of a tenant and drawing a document regarding that topology; (2) creating configuration commands for deploying that tenant; (3) validating the correctness of the commands (before inputting into devices); (4) crossvalidating those commands (performed by another engineer to avoid human-errors).

The use of the tool mitigated the burden of this operational procedure as follows.

- The tool achieved in reducing the operational time of the per-tenant network construction/validation from $240 \mathrm{~min}$. to $80 \mathrm{~min}$. The breakdown of the operational costs is displayed in Table 1. Configuration creation is fully automated by the template tool, whereas topology design still requires operational cost because of the need for manually creating documents including the drawings of their topologies. On the other hand, despite auto-generated configurations are correct and not needed to be validated in essence, configuration files generated by the tool are still cross-validated by the engineer to assure the correctness of the prototype.

- In addition to the above achievement, the tool contributed to reducing the number of dedicated engineers from 2 to 1 . The cross-validation requires another engineer in addition to the one in charge of creating configuration commands for the tolerance to human-error, needing at least 2 engineers in total. Since the template tool plays the role of configuration creator, there needs only 1 engineer for validation.
- The automation of deploying typical tenants enables engineers to concentrate their effort on the atypical (value-added) deals. This means that atypical cases could indirectly be accelerated by the template tool by mitigating the total operational cost on engineers.

In summary, the templates based on the discovered configuration patterns contributed to reducing operational time, human costs, and the possibility of human-error although not perfect yet.

\section{RELATED WORKS}

Configuration templates. A few works have developed configuration templates in different networking fields (e.g., ISP $[4,6,7])$, and the present work shares the spirit of the use of templates. In contrast to those work, an originality of this work is the focus on the fundamental and crucial difficulty in creating appropriate templates. Indeed, Refs. [10, 11] automatically find frequent device-level configuration patterns by means of a device-to-device copy-andpaste detection tool. However, this kind of tool cannot be directly applied to finding typical patterns of tenantlevel network-wide configuration, because tenant networks are structured and not constructed over a single network device. We instead select the approach of finding typical network-wide configuration by customizing a graph-mining technique according to the context of this problem. To the best of our knowledge, this is the first detailed statement on appropriately finding and using configuration templates in multi-tenant datacenter networks.

Network configuration analysis. There have been various efforts on analyzing network configuration files. Configuration files have been used mostly in understanding network characteristics such as routing design [12], Class-ofService [13], Route Redistribution [14], complexity of enterprise network [10], access control lists [15], co-occurrence of device updates [16], operational history [17], and longitudinal trends in ISP services [11] and campus networks [18]. Ref. [5] argues the possibility of the use of graph-mining on configuration files, but unfortunately does not provide details. This work newly develops the graph-mining-based method of analyzing configuration files.

Management in multi-tenant datacenters. In the context of device settings in multi-tenant datacenters, there are a few works on future datacenter architectures addressing management aspect as one of primary issues $[19,20]$. On the other hand, we focus on current situation where engineers use existing products of hardware and software commercially avaiable today, which new architectures of future paradigm cannot be directly applied. Existing tools (mostly commercial) provide intuitive user-friendly GUI interface for ease of settings, but such settings seem have to be made per instance of logical resource (e.g., VLAN, VRF), different from per-tenant deployment that we aim to achieve.

\section{DISCUSSION}

Generality. We consider that the proposed method would be applicable to a great deal of other multi-tenant datacenter networks, only if tenant networks are logically independent at the level of virtual resources ${ }^{1}$. Device-specific languages are abstracted as device-neutral graph components

\footnotetext{
${ }^{1}$ The proposed method cannot be directly applied to
} the case where separation among tenants are realized at 
(nodes/edges) for generality, and virtual resources defined in this work (VLAN, VIF, VR) are essential building-block of computer communications in principle. Configuration compositions of tenant networks are not necessarily specific to the analyzed datacenter, because a few design guides recommend similar (although not exactly same) patterns $[1,2]$. The effectiveness of the method would be impaired when all tenant networks are totally different, but this should be unrealistic in actual environments.

Limitation. The present work mainly focused on topological aspects of tenant networks, and did not capture access control functions such as routing, filtering (ACL), quality assurance (QoS). The identification of typical topologies should be the first step to discuss the capability and effectiveness of finding template regarding those access control functions, because they are performed over deployed topologies. Although the use of ACL has become less significant in multi-tenant datacenters (because the inter-tenant isolation is achieved by the logical independence of virtual resources), those remaining tasks would be interesting future works.

\section{CONCLUDING REMARKS}

We developed a configuration analysis method to automatically find appropriate (i.e., reusable) configuration templates in deploying tenant networks for virtualized multitenant datacenter networks. The main idea was based on mining existing tenant configurations to discover typical patterns that can be converted as reusable templates for upcoming tenants. The evaluation with actual configuration files obtained from a production datacenter network demonstrated the effectiveness of the proposed method. Future works include discovering domain-knowledge from configuration information in a systematic manner as well as quantifying the scalability characteristics of the method. Also, another interesting future work is to investigate the applicability of this method to multiple datacenters.

\section{REFERENCES}

[1] "Cisco Systems Inc., Cisco Virtualized Multi-Tenant Data Center, Version 2.1 Implementation Guide." [Online]. Available: http://www.cisco.com/en/US/docs/solutions/Enterprise/ Data_Center/VMDC/2.1/implementation_guide/vmdcImpl21.pdf

[2] "Juniper Networks Inc., Cloud Ready Data Center Network Design Guide." [Online]. Available: http://www.juniper.net/us/en/local/pdf/designguides/8020014-en.pdf

[3] A. Feldmann and J. Rexford, "IP Network Configuration for Intradomain Traffic Engineering," IEEE Network, vol. 15, pp. 46-57, September-October 2001.

[4] J. Gottlieb, A. Greenberg, J. Rexford, and J. Wang, "Automated provisioning of BGP customers," IEEE Network, vol. 17, pp. 44-55, November-December 2003.
[5] D. Caldwell, A. Gilbert, J. Gottlieb, A. Greenberg, G. Hjalmtysson, and J. Rexford, "The Cutting EDGE of IP Router Configuration," ACM SIGCOMM CCR, vol. 34, pp. 21-26, January 2004.

[6] W. Enck, P. McDaniel, S. Sen, P. Sebos, S. Spoerel, A. Greenberg, S. Rao, and W. Aiello, "Configuration Management at Massive Scale: System Design and Experience," in USENIX ATC'07, June 2007, pp. 73-86.

[7] L. Vanbever, G. Pardoen, and O. Bonaventure, "Towards validated network configurations with NCGuard," in IEEE INM'08, October 2008, p. 6.

[8] X. Gao, B. Xiao, D. Tao, and X. Li, "A survey of graph edit distance," Springer Pattern Analysis \& Applications, vol. 13, no. 1, pp. 113-129, February 2010.

[9] A. K. Jain, M. N. Murty, and P. J. Flynn, "Data clustering: a review," ACM Computing Surveys, vol. 31, no. 3, pp. 264-323, September 1999

[10] T. Benson, A. Akella, and D. Maltz, "Unraveling the Complexity of Network Management," in USENIX NSDI'09, April 2009, pp. 335-348.

[11] T. Benson, A. Akella, and A. Shaikh, "Demystifying Configuration Challenges and Trade-Offs in Network-based ISP Services," in ACM SIGCOMM'11, August 2011, pp. 302-313.

[12] D. Maltz, G. Xie, J. Zhan, H. Zhang, G. Hjalmtysson, and A. Greenberg, "Routing Design in Operational Networks: A Look from the Inside," in ACM SIGCOMM'04, August-September 2004, pp. 27-40.

[13] Y.-W. E. Sung, C. Lund, M. Lyn, S. Rao, and S. Sen, "Modeling and Understanding End-to-End Class of Service Policies in Operational Networks," in ACM SIGCOMM'09, August 2009, pp. 219-230.

[14] F. Le, G. G. Xie, D. Pei, J. Wang, and H. Zhang, "Shedding Light on the Glue Logic of the Internet Routing Architecture," in ACM SIGCOMM'08, August 2008, pp. 39-50.

[15] T. Benson, A. Akella, and D. Maltz, "Mining Policies From Enterprise Network Configuration," in $A C M I M C^{\prime} 09$, November 2009, pp. 136-142.

[16] Y.-W. E. Sung, S. Rao, S. Sen, and S. Leggett, "Extracting Network-Wide Correlated Changes from Longitudinal Configuration Data," in PAM'09, April 2009, pp. 111-121.

[17] D. Plonka and A. J. Tack, "An Analysis of Network Configuration Artifacts," in USENIX LISA'09, November 2009, p. 13.

[18] H. Kim, T. Benson, A. Akella, and N. Feamster, "The Evolution of Network Configuration: A Tale of Two Compuses," in ACM IMC'11, November 2011, pp. 499-512.

[19] A. Edwards, A. Fischer, and A. Lain, "Diverter: A New Approach to Networking Within Virtualized Infrastructures," in ACM WREN'09, August 2009, pp. 103-110.

[20] J. Mudigonda, P. Yalagandula, J. Mogul, B. Stiekes, and Y. Pouffary, "NetLord: A Scalable Multi-Tenant Network Architecture for Virtualized Datacenters," in $A C M$ SIGCOMM'11, August 2011, pp. 62-73.

application-level; In this case, the graph model has to additionally include the application-level information of separation, and the mining method will need to be extended to handle them, although the fundamental idea of this paper (i.e., mining per-tenant information) would be applied. 